\title{
ON THE EXPANSION OF ANALYTIC FUNCTIONS IN SERIES OF POLYNOMIALS*
}

BY

\section{J. L. WALSH}

\section{INTRODUCTION: STATEMENT OF PRINGIPAL METHOD AND RESULTS}

If $C$ is a closed contour in the plane of the complex variable $z$, there have been a number of proofs, the first of which was due to Runge, that any function $f(z)$ analytic on and interior to $C$ can be expanded in a series of polynomials in that region.t In particular it was shown by Faber that we may choose

$$
f(z)=a_{0} p_{0}(z)+a_{1} p_{1}(z)+\cdots+a_{n} p_{n}(z)+\cdots,
$$

where the polynomials $p_{k}(z)$ do not depend on the function $f(z)$ but merely on the curve $C$. The coefficients of the polynomials are given by the formulas

$$
a_{k}=\int_{C} f(z) P_{k}(z) d z
$$

where the functions $P_{k}(z)$ are properly chosen. The series (1) converges uniformly in the closed region interior to $C$.

This fundamental result is a direct generalization of Taylor's series, to which Faber's series (1) reduces when $C$ is a circle.

On any circle $C$ for which Taylor's series converges, if the center of $C$ is the point about which the Taylor development is considered, Taylor's series reduces precisely to Fourier's series, both formally and in fact. More generally, Laurent's series similarly reduces to Fourier's series and conversely, if the function considered is defined and integrable on the circle $C$. The natural generalization of Fourier's series and of Laurent's series to the case of an arbitrary contour $C$ seems not to have been made. It is the object of the present paper to set forth such a generalization, as indicated by the following theorem:

* Presented to the Society, December 27, 1923.

$\dagger$ Detailed references to the work of Runge and Faber are given by Montel, Leçons sur les Series à une Variable complexe, Paris, 1910. The method of conformal mapping used in $\$ 2$ of the present paper is of course well known. See for instance, Montel, chapter 3. 
Theorem I. Let $C$ be a simple closed finite analytic curve in the z-plane, including in its interior the origin. Then there exist two sets of functions

$$
\begin{array}{r}
p_{0}(z), p_{1}(z), \ldots, p_{n}(z), \ldots \\
q_{1}(z), \ldots, q_{n}(z), \ldots
\end{array}
$$

polynomials respectively in $z$ and $1 / z$, such that if $f(z)$ be any function defined on $C$ and satisfying on $C$ a Lipschitz condition, ${ }^{*}$ then $f(z)$ can be developed in the series

$$
\begin{aligned}
f(z)=a_{0} p_{0}(z) & +a_{1} p_{1}(z)+a_{2} p_{2}(z)+\cdots+a_{n} p_{n}(z)+\cdots \\
& +b_{1} q_{1}(z)+b_{\mathbf{2}} q_{2}(z)+\cdots+b_{n} q_{n}(z)+\cdots,
\end{aligned}
$$

where the former series converges uniformly in the closed region interior to $C$ and the latter series converges uniformly in the closed region exterior to $C$ and vanishes at infinity. $t$ The coefficients of (2) are given by the formulas

$$
a_{k}=\int_{C} f(z) s_{k}(z) d z, \quad b_{k}=\int_{C} f(z) t_{k}(z) d z
$$

where the functions $s_{k}(z)$ and $t_{k}(z)$ depend not on $f(z)$ but only on $C$. The functions $s_{k}(z)$ are analytic on and exterior to $C$ and vanish at infinity; the functions $t_{k}(z)$ are analytic on and interior to $C$. The polynomial $p_{k}(z)$ has precisely $k$ roots interior to $C$, and the polynomial $q_{k}(z)$ has precisely $k$ roots exterior to $C$.

It will be noted that this theorem differs from that of Faber in that (a) it considers the convergence of the series (2) on the curve $C$ itself, where $f(z)$ is not necessarily analytic on $C$, and $(b)$ it deals with functions $f(z)$ defined on $C$ but not necessarily analytic interior to $C$, expressing such functions as the sum of two series, the former convergent and representing a function analytic interior to $C$ and continuous in the closed region thus

* That is, there exists a constant $K$ such that the inequality

$$
\left|f\left(z_{1}\right)-f\left(z_{\mathbf{2}}\right)\right| \leqq K\left|z_{1}-z_{\mathbf{2}}\right|
$$

holds whatever may be the points $z_{1}$ and $z_{2}$ on $C$.

† This tacitly assumes that the functions $q_{k}(z)$ are defined to have the value zero at infinity, so that each of those functions is continuous in the closed region exterior to $C$. 
defined, the latter convergent and representing a function vanishing at infinity, analytic exterior to $C$, and continuous in the closed region thus defined. The writer is aware of no other treatment of this general problem involving either $(a)$ or $(b) .^{*}$

Let us briefly outline the proof of Theorem I before taking up the details of that proof. The region interior to $C$ can be mapped conformally on the interior of the unit circle $\gamma$ in the $w$-plane by the analytic mapping functions

$$
w=\varphi(z), \quad z=\psi(w) .
$$

Any function $f_{1}(z)$ analytic interior to $C$ is thus transformed into a function analytic interior to $\gamma$, and in the interior of $\gamma$ can be expanded in powers of $w$. If the function $f_{1}(z)$ satisfies a Lipschitz condition on $C$ (or on $\gamma$ ), this development is valid also on $\gamma$ itself. That is, in and on $C$, the function $f_{1}(z)$ can be expanded in terms of the powers of $\varphi(z)$

$$
1, \varphi(z), \varphi^{2}(z), \ldots, \varphi^{n}(z), \ldots
$$

The set of functions (4) can be replaced by functions which do not differ greatly from them, without altering the essential convergence properties of the set.t In particular we may choose a set

$$
p_{0}(z), p_{1}(z), p_{\mathbf{2}}(z), \ldots, p_{n}(z), \ldots
$$

of polynomials, for within and on $C$ any function of the set (4) can be uniformly approximated by a polynomial.

In precisely the same manner, the region exterior to $C$ may be mapped on the unit circle $\gamma$, and we find a set of polynomials in $1 / z$,

$$
q_{1}(z), q_{2}(z), \ldots, q_{n}(z), \ldots,
$$

* The results of Faber can be extended by considering simultaneously the interior and exterior regions, using the methods of $\S \S 4$ and 5. That treatment has the advantage over the present treatment of giving definite regions of convergence and of divergence for the series in (2) in every case, the regions depending on the singularities of the analytic functions represented by those series. That treatment has the disadvantage of requiring (for application of Faber's results) the consideration only of functions analytic on $C$.

The same remark obtains for the results of Szegö, Mathematische Zeitschrift, vol. 9 (1921), pp. 218-270.

$\dagger$ If the mapping function $\varphi(z)$ is a polynomial, we may set

$$
p_{k}(z)=\varphi^{k}(z) \text {. }
$$

A similar remark holds for the functions $q_{k}(z)$. 
in terms of which there can be developed any function $f_{\mathbf{z}}(z)$ which satisfies a Lipschitz condition on $C$, is analytic exterior to $C$, and vanishes at infinity. By a theorem due to Plemelj, any function $f(z)$ defined on $C$ and there satisfying a Lipschitz condition can be expressed on $C$ in the form

$$
f(z)=f_{1}(z)+f_{\mathbf{2}}(z),
$$

where $f_{1}$ and $f_{2}$ are functions of the kind required for that notation. Thus $f(z)$ can be expanded in terms of the two sets (5) and (6), and if the functions $s_{k}(z)$ and $t_{k}(z)$ are properly chosen the coefficients are given by (3), and the theorem is established.

We proceed to the details of the proof.

\section{EXPansion in terms OF MAPPING FUNCTION AND ITS POWERS}

The contour $C$ has been assumed analytic, so its interior can be mapped on the unit circle $\gamma$ in the $w$-plane:

$$
w=\varphi(z),
$$

the inverse transformation being

$$
z=\psi(w) .
$$

We suppose the origins in the two planes to correspond:

$$
\varphi(0)=0, \quad \psi(0)=0 .
$$

The function $\psi^{\prime}(w)$ is analytic not merely in the circle $\gamma:|w|=1$, but also on and within a larger circle $\gamma^{\prime}:|w|=1+\varepsilon$. We can and do choose the positive number $\varepsilon$ so small that the circle $\gamma^{\prime}$ corresponds in the $z$-plane to a simple analytic closed curve $C^{\prime}$ which surrounds the curve $C$.

Let $f_{1}(z)$ be any function which satisfies on $C$ a Lipschitz condition and is analytic interior to $C$. Then $f_{1}[\psi(w)]$ satisfies a Lipschitz condition on $\gamma$, so we have on and within $\gamma$ the series

$$
f_{1}[\psi(w)]=\sum_{n=0}^{\infty} a_{n} w^{n}, \quad a_{n}=\frac{1}{2 \pi i} \int_{\gamma} \frac{f_{1}[\psi(w)] d w}{w^{n+1}} .
$$


This series converges uniformly on $\gamma$, and hence in the closed region consisting of $\gamma$ and its interior. We have on and within $C$ the same series uniformly convergent in the closed region:

$$
f_{1}(z)=\sum_{n=0}^{\infty} a_{n}[\varphi(z)]^{n}, \quad a_{n}=\frac{1}{2 \pi i} \int_{C} \frac{f_{1}(z) \varphi^{\prime}(z) d z}{[\varphi(z)]^{n+1}}
$$

The set of functions (4), in terms of which $f_{1}(z)$ has been developed, is now to be replaced by a new set of functions

\section{ON THE EQUIVALENCE OF EXPANSIONS}

We shall find it convenient to prove, for later application, the following theorem:

THEOREM II. Let the functions

$$
p_{n}(x), p_{1}(x), \ldots, p_{n}(x), \ldots
$$

be analytic for $|x| \leqq 1+\varepsilon$, and such that on and within the circle $\gamma^{\prime}$, $|x|=1+\varepsilon$, we have

$$
\left|p_{k}(x)-x^{k}\right| \leqq \varepsilon_{k} \quad(k=0,1,2, \ldots) .
$$

where the series $\sum \varepsilon_{k}^{2}$ converges to a sum less than unity, and where the series $\sum \varepsilon_{\boldsymbol{k}}$ converges. Then any function $F(z)$ which is continuous for $|x| \leqq 1$, analytic for $|x|<1$ and which on the circle $\gamma,|x|=1$, satisfies a Lipschitz condition, can be developed into a series

$$
F(x)=\sum_{k=0}^{\infty} c_{k} p_{k}(x)
$$

which converges uniformly for $|x| \leqq 1$.

There exists a set of functions $P_{k}(x)$ such that the coefficients of $(10)$ arc given by

$$
c_{k}=\int_{\gamma} F(x) P_{k}(x) d x .
$$

The functions $P_{k}(x)$ are analytic for $|x| \geqq 1$ and vanish at infinity. 
Theorem II is practically identical with a theorem due to Birkhoff,* but differs from that theorem slightly in the nature on $\gamma$ of the function $F(z)$ considered. We prove Theorem II by means of a lemma; in the statement of this lemma the symbol $\delta_{n k}$ is the Kronecker symbol which has the value zero or unity according as $n$ and $k$ are or are not distinct.

Lemma. Suppose that $\left\{u_{n}(\varphi)\right\}$ is a set of uniformly bounded normal orthogonal functions in the interval $0 \leqq \varphi \leqq 2 \pi$ :†

$$
\int_{0}^{2 \pi} u_{n}(\varphi) \bar{u}_{k}(\varphi) d \varphi=\delta_{n k} \quad(n, k=0,1,2, \ldots)
$$

and that in this interval $\left\{U_{n}(\varphi)\right\}$ is a set of uniformly bounded continuous functions each of which can be developed into a series

$$
U_{n}=\sum_{k=0}^{\infty}\left(c_{n k}+\delta_{n k}\right) u_{k} \quad(n, k=0,1,2, \ldots)
$$

where the coefficients have the values

$$
c_{n k}+\delta_{n k}=\int_{0}^{2 \pi} U_{n} \bar{u}_{k} d \varphi .
$$

Suppose further that the three series

$$
\sum_{n, k=0}^{\infty} c_{n k} \overline{c_{n k}}, \quad \sum_{n=0}^{\infty}\left(\sum_{k=0}^{\infty} c_{n k} \overline{c_{n k}}\right)^{\frac{1}{2}}, \quad \sum_{k=0}^{\infty}\left(\sum_{n=0}^{\infty} c_{n k} \overline{c_{n k}}\right)^{\frac{1}{2}}
$$

converge and that the value of the first is less than unity.

Then there exists a set of continuous functions $\left\{V_{n}(\varphi)\right\}$ such that $\left\{U_{n}\right\}$ and $\left\{V_{n}\right\}$ are biorthogonal sets:

$$
\int_{0}^{2 \pi} U_{n} \bar{V}_{k} d \varphi=\delta_{n k} \quad(n, k=0,1,2, \ldots) .
$$

* Paris Comptes Rendus, vol. 164 (1917), pp. 942-945. The lemma used in proving Theorem II was given by Walsh, these Transactions, vol. 22 (1921), p. 230-239. The proof of the lemma was there given for the real case, but extends without difficulty to the complex case. For our present application $\left\{u_{n}\right\}$ is real, while $\left\{U_{n}\right\}$ is not.

$\dagger$ The dash here indicates the conjugate of the complex quantity beneath. 
Furthermore, if $f(\varphi)$ is any function integrable and with an integrable square (in the sense of Lebesgue), then the two series

$$
f(\varphi) \sim \sum_{n=0}^{\infty} \alpha_{n} u_{n}(\varphi), \quad f(\varphi) \sim \sum_{n=0}^{\infty} \beta_{n} U_{n}(\varphi)
$$

where

$$
\alpha_{n}=\int_{0}^{2 \pi}\left(f(\varphi) \overline{u_{n}(\varphi)} d \varphi, \quad \beta_{n}=\int_{0}^{2 \pi} f(\varphi) \overline{V_{n}(\varphi)} d \varphi\right.
$$

have essentially the same convergence properties.

The sign $\sim$ is used simply to indicate that the coefficients $\alpha_{n}$ and $\beta_{n}$ are given by (18), which must be the case if the series converge uniformly to the value $f(\varphi)$. The two series are said to have essentially the same convergence properties when and only when the series

$$
\sum_{n=0}^{\infty}\left(\alpha_{n} u_{n}-\beta_{n} U_{n}\right)
$$

converges absolutely and uniformly to the sum zero, no matter what may be the function $f(\varphi)$ considered.

If any function $F(\varphi)$ is integrable and has an integrable square on the interval $0 \leqq \varphi \leqq 2 \pi$, we have the result

$0 \leqq \int_{0}^{2 \pi}\left(F-\gamma_{0} u_{0}-\gamma_{1} u_{1}-\cdots-\gamma_{n} u_{n}\right)\left(\bar{F}-\bar{\gamma}_{0} \bar{u}_{0}-\bar{\gamma}_{1} \bar{u}_{1}-\cdots-\bar{\gamma}_{n} \bar{u}_{n}\right) d \varphi$, or, if $\gamma_{k}=\int_{0}^{2 \pi} F \bar{u}_{k} d \varphi$, we have

$$
\int_{0}^{2 \pi} F \bar{F} d \varphi \geqq \gamma \bar{\gamma}_{0}+\gamma_{1} \bar{\gamma}_{1}+\cdots+\gamma_{n} \bar{\gamma}_{n}
$$

There are a number of steps to be taken in applying the lemma to the proof of Theorem II. The interval $0 \leqq \varphi \leqq 2 \pi$ is to be chosen as the circle $\gamma,|x|=1$, using $x=e^{i \varphi}$ on $\gamma$. The functions $\left\{u_{n}(\varphi)\right\}$ and $\left\{U_{n}(\varphi)\right\}$ are to be chosen as 


$$
\begin{aligned}
& u_{0}=\frac{1}{\sqrt{2 \pi}}, \\
& U_{0}=\frac{1}{\sqrt{2 \pi}} p_{0}(x) \\
& u_{1}=\frac{1}{2 \sqrt{\pi}}\left(x+\frac{1}{x}\right) \text {, } \\
& U_{1}=\frac{1}{2 \sqrt{\pi}}\left[p_{1}(x)+\frac{1}{x}\right], \\
& u_{2}=\frac{i}{2 \sqrt{\pi}}\left(x-\frac{1}{x}\right) \text {, } \\
& U_{2}=\frac{i}{2 \sqrt{\pi}}\left[p_{1}(x)-\frac{1}{x}\right] \text {, } \\
& u_{3}=\frac{1}{2 \sqrt{\pi}}\left(x^{2}+\frac{1}{x^{2}}\right) \text {, } \\
& U_{3}=\frac{1}{2 \sqrt{\pi}}\left[p_{2}(x)+\frac{1}{x^{2}}\right] \text {, } \\
& u_{4}=\frac{i}{2 \sqrt{\pi}}\left(x^{2}-\frac{1}{x^{2}}\right), \\
& U_{4}=\frac{i}{2 \sqrt{\pi}}\left[p_{8}(x)-\frac{1}{x^{2}}\right] \text {, } \\
& u_{2 n-1}=\frac{1}{2 \sqrt{\pi}}\left(x^{n}+\frac{1}{x^{n}}\right), \\
& U_{2 n-1}=\frac{1}{2 \sqrt{\pi}}\left[p_{n}(x)+\frac{1}{x^{n}}\right] \text {, } \\
& u_{2 n}=\frac{i}{2 \sqrt{\pi}}\left(x^{n}-\frac{1}{x^{n}}\right), \\
& U_{2 n}=\frac{i}{2 \sqrt{\pi}}\left[p_{n}(x)-\frac{1}{x^{n}}\right],
\end{aligned}
$$

The two sets of functions $\left\{u_{n}\right\}$ and $\left\{U_{n}\right\}$ are obviously uniformly bounded and continuous on the interval considered. The functions $\left\{U_{n}\right\}$ are analytic on $\gamma$ and hence can be developed on $\gamma$ in the series (13). By inequality (19) for the function $F=U_{n}-u_{n}$ we have

$$
\begin{aligned}
& c_{n k}=\int_{0}^{2 \pi}\left(U_{n}-u_{n}\right) \bar{u}_{k} d \varphi, \\
& \sum_{k=0}^{\infty} c_{n k} \overline{c_{n k}} \leqq \int_{0}^{2 \pi}\left(U_{n}-u_{n}\right)\left(\overline{U_{n}}-\bar{u}_{n}\right) d \varphi \leqq\left\{\begin{array}{l}
\varepsilon_{0}^{2}, n=0 \\
\frac{\varepsilon_{m}^{2}}{2}, n \neq 0\left\{\begin{array}{l}
m=\frac{n}{2}, n \text { even } \\
m=\frac{n+1}{2}, n \text { odd }
\end{array}\right.
\end{array}\right.
\end{aligned}
$$

Thus the first of series (15) converges and its sum is less than unity. The convergence of $\sum_{k=c}^{\infty}{ }_{k}$ gives us the convergence of the second of the series (15). To study the third of those series we make use of the fact that the functions $p_{k}(x)$ are all analytic on and within the circle $\gamma^{\prime}$. Thus we have 


$$
\begin{aligned}
c_{n k}=\int_{0}^{2 \pi}\left(U_{n}-u_{n}\right) \bar{u}_{k} d \varphi & = \pm \frac{i^{n+k}}{4 \pi} \int_{\gamma}\left[p_{m}(x)-x^{m}\right]\left[x^{l} \pm \frac{1}{x^{l}}\right] \frac{d x}{i x} \\
& = \pm \frac{i^{n+k}}{4 \pi i} \int_{\gamma^{\prime}}\left[p_{m}(x)-x^{m}\right] \frac{d x}{x^{l+1}} \\
m, l>0, l & =\left\{\begin{array}{l}
\frac{k}{2}, k \text { even, } \\
\frac{k+1}{2}, k \text { odd, }
\end{array} \quad m=\left\{\begin{array}{l}
\frac{n}{2}, n \text { even, } \\
\frac{n+1}{2}, n \text { odd. }
\end{array}\right.\right.
\end{aligned}
$$

Then we find

$$
\left|c_{n k}\right| \leqq \frac{\varepsilon_{m}}{(1+\varepsilon)^{l}}
$$

The case $m, l=0$ is readily disposed of, and yields also inequality (20). The convergence of the third of the series (15) now presents no further difficulty.

It follows from the form of the functions $\left\{u_{n}\right\}$ and $\left\{U_{n}\right\}$ that the series (13) and likewise (17) can be written by combining terms so that negative powers of $x$ are eliminated, if $f(\varphi)$ is equal to the function $F(x)$ of Theorem II. Thus the second of series (17) can be identified with (10).

Theorem II is now completely proved except for the remark concerning the functions $P_{k}(x)$. If the proof of the Lemma is examined, it will be seen that the series for $P_{k}(x)$ converge uniformly in the neighborhood of the circle $\gamma,{ }^{*}$ and from the special form of the functions $U_{n}(x)$ that we are considering it follows that the analytic functions $P_{k}(x)$ thus defined are analytic on and everywhere outside of $\gamma$ and vanish at infinity.

\section{Choice of polynomials}

We return now to the set of functions (4), and shall replace the set by a new set consisting of polynomials. By the theorem of Runge we can uniformly approximate to the function $\varphi^{k}(z)$ as closely as desired in the closed region interior to $C^{\prime}$ by a polynomial, for $\varphi^{k}(z)$ is analytic in that

* Loc. cit., p. 234. We shall have, in our present notation,

$$
V_{k}=\sum_{n=0}^{\infty}\left(d_{k n}+\delta_{k n}\right) u_{n}
$$

and we have from the definition of the $d_{m n}$, from (20) and from the inequality (11) of the other paper, that a geometric series dominates this series for $\nabla_{k}$. 
closed region. Let us choose a set of numbers $\varepsilon_{0}, \varepsilon_{1}, \varepsilon_{2}, \ldots$ satisfying the requirement of Theorem II and then determine polynomials $p_{k}(z)$ so that all the inequalities

$$
\left|p_{k}(z)-\varphi^{k}(z)\right| \leqq \varepsilon_{k}
$$

are satisfied on and interior to $C^{\prime}$.

If the numbers $\varepsilon_{k}$ are chosen sufficiently small, the polynomial $p_{k}(z)$ will have precisely $k$ roots interior to $C^{\prime}$, and interior to $C$. For on either $C$ or $C^{\prime}$ we have*

$$
p_{k}(z)=\varphi^{k}(z)\left[\frac{p_{k}(z)}{\varphi^{k}(z)}\right]
$$

The last factor is practically equal to unity, and by suitable choice of $\varepsilon_{k}$ can be made as near to unity as desired, uniformly in the closed region between $C$ and $C^{\prime}$. Thus when either of the contours $C$ or $C^{\prime}$ is traced, the total increase in the argument of the complex quantity $p_{k}(z)$ is the same as the total increase in the argument of $\varphi^{k}(z)$.

We may choose $p_{k}(z)$ so that for any particular value of $k$ or for all values of $k$ these $k$ roots interior to $C$ are distinct or coincident, at pleasure. For to cause them to coincide, choose a polynomial $\pi_{k}(z)$ such that

$$
\left|\pi_{k}(z)-\varphi(z)\right| \leqq \frac{\varepsilon_{k}^{\prime}}{2}, \text { where } \varepsilon_{k}^{\prime} \leqq M, \frac{\varepsilon_{k}}{2^{k-1} k M^{k-1}},|\varphi(z)| \leqq M
$$

Then since $\varphi(0)=0$, we have

$$
\begin{aligned}
& \left|\left[\pi_{k}(z)-\pi_{k}(0)\right]-\varphi(z)\right| \leqq \epsilon_{k}^{\prime}, \\
& \quad\left[\pi_{k}(z)-\pi_{k}(0)\right]^{k}-\varphi^{k}(z) \\
& \quad=\left[\pi_{k}(z)-\pi_{k}(0)-\varphi(z)\right]\left\{\left[\pi_{k}(z)-\pi_{k}(0)\right]^{k-1}+\cdots+\varphi^{k-1}(z)\right\},
\end{aligned}
$$

so the polynomial

$$
p_{k}(z)=\left[\pi_{k}(z)-\pi_{k}(0)\right]^{k}
$$

has the property required. To cause the $k$ roots of $p_{k}(z)$ in $C$ to remain distinct, alter slightly the coefficients of the particular polynomial $p_{k}(z)$

* This result also follows from a general theorem due to Hurwitz, Mathematische Annalen, vol. 33 (1888), p. 248. 
just considered so that its discriminant does not vanish, yet so that we still satisfy the inequality

$$
\left|p_{k}(z)-\varphi^{k}(z)\right| \leqq \varepsilon_{k}
$$

use a new $\varepsilon_{k}^{\prime}$ if necessary.

We apply now Theorem II to the polynomials $p_{k}(z)$, or rather to their transforms in the $w$-plane. The functions $1, w, w^{2}, \ldots$ expand any function $F(w)$ analytic interior to $\gamma$ and satisfying a Lipschitz condition on $\gamma$ itself; the resulting series converges uniformly in the closed region consisting of $\gamma$ and its interior. The set of functions $p_{k}[\psi(w)]$ likewise expands any such function $F(w)$; the resulting series converges uniformly on $\gamma$, by virtue of Theorem II, and hence converges uniformly in the entire closed region consisting of $\gamma$ and its interior. This series is

$$
F(w)=\sum_{k=0}^{\infty} c_{k} p_{k}[\psi(w)], \quad c_{k}=\int_{\gamma} F(w) P_{k}(w) d w
$$

We have the corresponding formulas in the $z$-plane,

$$
F[\varphi(z)]=\sum_{k=0}^{\infty} c_{k} p_{k}(z), \quad c_{k}=\int_{C} F[\varphi(z)] P_{k}[\varphi(z)] \varphi^{\prime}(z) d z
$$

where the series converges uniformly on $C$ and hence uniformly in the closed region consisting of $C$ and its interior.

The function $P_{k}[\varphi(z)] \varphi^{\prime}(z)$ is analytic on the curve $C$, and hence on that curve may be expressed as the sum of two functions, of which the first is analytic on and interior to $C$, and the second analytic on and exterior to $C$ and vanishes at infinity.* The former function gives no contribution to the integral (22) for $c_{k}$, no matter what may be the function $F(z)$ analytic interior to $C$. Then we may and do replace $P_{k}[\varphi(z)] \varphi^{\prime}(z)$ by the latter of the two functions, which is denoted by $s_{k}(z)$. We replace the formula of (22) by

$$
c_{k}=\int_{C} F[\varphi(z)] s_{k}(z) d z .
$$

\footnotetext{
* This resolution is set up immediately by Cauchy's integral formula applied to a closed ring-shaped region in which the function considered is analytic, the region bounded by two simple closed curves and containing $C$ in its interior.
} 
We notice that if there is substituted formally in the integral of (23) any function $f_{\mathbf{2}}(z)$ which is continuous on and exterior to $C$, analytic exterior to $C$, and which vanishes at infinity, then the resulting integral is zero:

$$
\int_{C} f_{\mathbf{Z}}(z) s_{k}(z) d z=0 .
$$

Whenever the function $F[\varphi(z)]$ satisfies a Lipschitz condition on $C$, then $F(w)$ satisfies a Lipschitz condition on $\gamma$, and hence the series development (22) converges uniformly on and interior to $C$, to the sum $F[\varphi(z)]$.

\section{Problem in INNER AND OUter Regions}

We have thus proved the possibility of expanding in a series of type (22) any function $f_{1}(z)$ of the kind described. By the same methods, mapping the exterior of $C$ on the interior of the unit circle so that the point at infinity corresponds to the origin, we can find a set $q_{k}(z)$ of polynomials in $1 / z$ in terms of which there can be expanded any function $f_{2}(z)$ analytic exterior to $C$, vanishing at infinity, and satisfying on $C$ a Lipschitz condition:

$$
f_{\mathbf{2}}(z)=\sum_{k=1}^{\infty} b_{k} q_{k}(z), \quad b_{k}=\int_{C} f_{\mathbf{2}}(z) t_{k}(z) d z .
$$

The series (25) converges uniformly throughout the closed region consisting of $C$ and its exterior.

It is to be noted that in (25) we have omitted the term $b_{0} q_{0}(z)$. It is possible to do this, for we choose $q_{0}(z)$ equal to unity, $q_{k}(\infty)=0$ for $k>0$. Then it follows from the series for the functions $t_{k}(z)$ that $b_{0}$ vanishes whenever $f_{\mathbf{z}}(z)$ vanishes at infinity.

The functions $t_{k}(z)$ are analytic on $C$, and hence on $C$ can be expressed as the sum of two functions, of which the first is analytic on and interior to $C$, and the second is analytic on and exterior to $C$ and vanishes at infinity. The latter component of $t_{k}(z)$ gives no contribution to the integral (25), no matter what may be the function $f_{z}(z)$ satisfying the prescribed conditions. We may therefore replace each function $t_{k}(z)$ by its first component, which we do without change of notation. Formulas (25) still hold, and we also have, if $f_{1}(z)$ is analytic interior to $C$ and continuous in the closed region thus formed,

$$
\int_{C} f_{1}(z) t_{k}(z) d z=0 .
$$


Suppose now that $f(z)$ is any function defined on $C$ and satisfying on $C$ a Lipschitz condition. Then on $C$ we may write*

$$
f(z)=f_{1}(z)+f_{\mathbf{2}}(z),
$$

where $f_{1}(z)$ is analytic interior to $C$, continuous on and interior to $C$, and satisfies a Lipschitz condition on $C$, and where $f_{\mathbf{z}}(z)$ is analytic exterior to $C$, vanishes at infinity, is continuous exterior to and on $C$, and satisfies on $C$ a Lipschitz condition. Then we have the expansions

$$
\begin{aligned}
& f_{1}(z)=a_{0} p_{0}(z)+a_{1} p_{1}(z)+\cdots+a_{n} p_{n}(z)+\cdots, a_{k}=\int_{C} f_{1}(z) s_{k}(z) d z . \\
& f_{\mathbf{z}}(z)=b_{1} q_{1}(z)+b_{\mathbf{2}} q_{\mathbf{2}}(z)+\cdots+b_{n} q_{n}(z)+\cdots, b_{k}=\int_{C} f_{\mathbf{Z}}(z) t_{k}(z) d z,
\end{aligned}
$$

where the series converge uniformly in the closed regions respectively interior and exterior to $C$. It follows from formulas (24), (26), (27) that these series give us the development (2) and formulas (3).

* By virtue of a theorem due to Plemelj, Monatshefte für Mathematik und Physik, vol. 19 (1908), pp. 205-210. See also Birkhoff, Proceedings of the American Academy of Arts and Sciences, vol. 49 (1913), pp. 521-568.

It can be shown that $f_{1}(z) \equiv 0$ if and only if

and $f_{2}(z) \equiv 0$ if and only if

$$
\int_{C} f(z) z^{n} d z=0 \quad(n=-1,-2,-3, \ldots),
$$

$$
\int_{C} f(z) z^{n} d z=0 \quad(n=0,1,2, \ldots) .
$$

See Walsh, Paris Comptes Rendus, vol. 178 (1924), pp. 58-59. This last result easily gives us the uniqueness of the resolution of $f(z)$ indicated in (27).

The conditions just given are respectively equivalent to the conditions

$$
\int_{C} f(z) s_{k}(z) d z=0, \quad \int_{C} f(z) t_{k}(z) d z=0,
$$

in the notation of Theorem I. 


\section{SOME OTHER POLYNOMIAL DEVELOPMENTS}

The requirement of Theorem $I$ that the given function satisfy on $C$ a Lipschitz condition is not necessary for expansion in a series of polynomials, if the function given on $C$ is the continuous boundary values taken on by an analytic function. In fact, Runge's theorem (or Theorem I) can be applied to prove the following result:*

Theorem III. Let $C$ be a finite simple analytic closed curve in the plane of the complex variable $z$. If $f_{1}(z)$ is a function of $z$ analytic interior to $C$ and continuous in the closed region consisting of $C$ and its interior, then $f_{1}(z)$ can be expanded in a series of polynomials

$$
f_{1}(z)=\pi_{1}(z)+\left(\pi_{2}(z)-\pi_{1}(z)\right)+\left(\pi_{3}(z)-\pi_{2}(z)\right)+\cdots,
$$

and the series converges uniformly in the closed region consisting of $C$ and its interior.

The writer is aware of no general result (other than Theorem I) which does not require analyticity in the closed region consisting of $C$ and its interior to establish the uniform convergence of (28) in that closed region.

The transformations

$$
w=\varphi(z), \quad z=\psi(w)
$$

already considered, map the interior of $C$ on the interior of the unit circle $\gamma$ in the $w$-plane. Form a sequence of circles

$$
\gamma_{1}, \gamma_{2}, \gamma_{3}, \ldots
$$

* Theorem III generalizes Theorem I merely in the case corresponding to $f_{2}(z) \equiv 0$. In this same case, an obvious application of the method used in the proof of Theorem III, without the use of conformal transformation, extends Theorem III to the case that $C$ is any contour which is either convex or convex with respect to a particular point $O$ interior to $C$; that is, a contour $C$ such that no half-line terminating at $O$ cuts $C$ in more than one point.

If the general function $f(z)$ of Theorem I is known merely to be continuous instead of satisfying a Lipschitz condition on $C$, then $f(z)$ can be uniformly approximated on $C$ by functions which are analytic on $C$. Each of these latter functions can by Theorem $I$ be uniformly approximated on $C$ by a rational function of $z$ which is the sum of a polynomial in $z$ and a polynomial in $1 / z$. Thus $f(z)$ can be expressed on $C$ as a uniformly convergent series of rational functions of $z$; each of these rational functions may be chosen as the sum of a polynomial in $z$ and a polynomial in $1 / z$. This is analogous to the theorem that any real function continuous in a closed interval can be expressed in that interval as the sum of a uniformly convergent series of trigonometric functions. 
all exterior to $\gamma$, interior to the circle $\gamma^{\prime}$ previously considered, which have the origin as their common center, and whose respective radii

$$
r_{1}, r_{2}, r_{3}, \ldots
$$

approach the limit unity. These circles correspond to simple analytic closed curves in the $z$-plane

$$
C_{1}, \quad C_{2}, \quad C_{3}, \ldots,
$$

each of which contains $C$ in its interior and $C^{\prime}$ in its exterior.

The function

$$
F(w) \equiv f_{1}[\psi(w)]
$$

is analytic in the region interior to $\gamma$ and continuous in the closed region consisting of $\gamma$ and its interior. The functions

$$
F_{k}(w) \equiv F\left(\frac{w}{r_{k}}\right) \quad(k=1,2,3, \ldots)
$$

are analytic respectively in the interiors of the regions

$$
\gamma_{1}, \gamma_{2}, \gamma_{3}, \ldots
$$

and are continuous in the corresponding closed regions. Moreover, since $F(w)$ is continuous in the closed region consisting of $\gamma$ and its interior, the sequence

$$
\left\{F_{k}(w)\right\}
$$

converges to the limit $F(w)$ uniformly on $\gamma$ and hence in the closed region consisting of $\gamma$ and its interior. Thus, whenever $\eta_{k}$ is given, we can choose $k$ so that on and within $\gamma$ we have

$$
\left|F_{k}(w)-F(w)\right|<\frac{\eta_{k}}{2} ;
$$

on and within $C$ we have

$$
\left|F_{k}[\varphi(z)]-f_{1}(z)\right|<\frac{\eta_{k}}{2} .
$$


The function $F_{k}(w)$ is analytic throughout the interior of $\gamma_{k}$, so the function

$$
F_{k}[\varphi(z)]
$$

is analytic throughout the interior of $C_{k}$. Then by Runge's theorem we can find a polynomial $\pi_{k}(z)$ such that on and within $C$ we have

$$
\left|\pi_{k}(z)-F_{k}[\varphi(z)]\right|<\frac{\eta_{k}}{2}
$$

It is now clear from (29) and (30) that we can choose a sequence of polynomials $\pi_{k}(z)$ convergent to the limit $f_{1}(z)$ uniformly throughout the closed region consisting of the curve $C$ and its interior.

In the series expansion (28) for $f_{1}(z)$ we do not of course have (even if $C$ is a circle) the polynomials which are the terms of (28) independent, except for a constant factor, of the function $f_{1}(z)$.

HARVARD UNIVTRSITY,

Cambridge, Mass. 